\title{
Síndrome metabólica em militares de uma unidade da polícia de Aracaju, Sergipe
}

\author{
Metabolic syndrome among police officers in a police station \\ from the city of Aracaju, Sergipe State, Brazil
}

\author{
Ilva Santana Santos Fontes ${ }^{1,2} \bowtie$, Clarissa Gomes Sampaio', Carla Geane dos Santos ${ }^{1}$, Angela Maria Melo Sá Barros ${ }^{1}$, \\ Diego Santos Fontes ${ }^{1}$, Irlan Massai Calaça dos Santos ${ }^{1}$, Luciano Jorge dos Santos Junior ${ }^{1}$ \\ ${ }^{1}$ Curso de Enfermagem da Universidade Tiradentes (UNIT). Aracaju, SE. \\ ${ }^{2}$ Curso de Enfermagem da Universidade Federal de Sergipe (UFS). Aracaju, SE.
}

\section{RESUMO}

Objetivos: Determinar a prevalência de síndrome metabólica e de seus componentes nos militares de uma unidade da polícia em Aracaju, Sergipe.

Métodos: Foi realizado um estudo transversal envolvendo os policiais militares de uma unidade de polícia localizada em Aracaju, capital do estado de Sergipe, no período de junho a julho de 2016. Foram selecionados para o estudo todos os policiais ativos na unidade que aceitaram participar da pesquisa, assinaram o termo de consentimento e não estavam em licença, afastamento, férias, ou processo de transferência para outra unidade. Para a coleta dos dados foi utilizado um questionário com informações sociodemográficas e uma ficha com dados antropométricos e de exames laboratoriais realizados pelos militares por ocasião do exame médico periódico de rotina. A síndrome metabólica foi definida a partir dos critérios do National Cholesterol Education Program 's Adult Treatment Panel III. Uma análise univariada foi realizada para examinar associações entre as variáveis de interesse, utilizando-se o teste qui-quadrado de Pearson e adotando-se o valor de $\mathrm{p}<0,05$ como estatisticamente significativo. Resultados: No período do estudo 115 policiais militares estavam lotados na unidade em estudo e $96(83,5 \%)$ entre eles preencheram os critérios de inclusão no estudo. Destes, a maioria era do sexo masculino (96,6\%), com filhos $(84,4 \%)$, casados $(52,2 \%)$, apresentavam nível de escolaridade até o ensino médio $(35,4 \%)$, autorreferiram-se pardos $(61,5 \%)$, ganhavam entre cinco e seis salários mínimos mensais $(42,7 \%)$, tinham idade entre 36 e 45 anos $(59,1 \%)$ e eram soldados $(53,1 \%)$. Um total de sete $(7,3 \%)$ entre os participantes do estudo apresentavam síndrome metabólica. Em relação aos componentes da síndrome metabólica, identificou-se circunferência abdominal aumentada indicando risco para doenças cardiovasculares em 34,4\% dos participantes; sobrepeso em 57,3\%; hipertensão arterial em 11,5\%; triglicerídeos elevados em 29,5\%; baixo colesterol ligado a lipoproteínas de alta densidade em 60,4\%; e glicemia de jejum elevada em 31,3\%. A graduação de cabo apresentou associação com síndrome metabólica: um militar com graduação de cabo tinha risco em torno de onze vezes mais de ter critérios para síndrome metabólica em relação às outras graduações (razão de prevalência=11,86; intervalo de confiança 95\% 10,88-12,84; p=0,005). Conclusões: Os policiais da amostra investigada apresentaram uma prevalência de síndrome metabólica menor quando comparada a outros estudos brasileiros. A graduação de cabo apresentou maior risco de desenvolver síndrome metabólica, o que deve ser investigado com mais profundidade por meio de estudos que relacionem as características funcionais de cada patente com a presença dos componentes clínicos e laboratoriais da síndrome metabólica.

DESCRITORES: síndrome metabólica; obesidade; resistência a insulina; pressão arterial; risco cardiovascular; policiais militares.

\section{ABSTRACT}

Aims: To assess the prevalence of metabolic syndrome and its components in the military officers of a police unit in Aracaju, Sergipe.

Methods: A cross-sectional study involving a military police unit located in Aracaju, capital of Sergipe state, Brazil, was conducted in the period June-July 2016. All active military policemen in the unit who agreed to participate were selected for the study. To participate, they were asked to sign the consent form and should not be on leave, departure, vacation, or transfer process to another unit. For data collection a questionnaire with demographic information was used, and a form was filled with anthropometric data and results of laboratory tests carried out during the medical routine periodic review. Metabolic syndrome was defined according to criteria of the National Cholesterol Education Program's Adult Treatment Panel III. Univariate analysis was performed to examine associations between the variables of interest, using the Pearson's chi-square test and adopting the value of $\mathrm{p}<0.05$ as statistically significant.

Results: During the study period 115 military policemen were stationed in the unit under study and $96(83.5 \%)$ among them met the criteria for inclusion in the study. Of these, the majority were male $(96.6 \%)$ with children $(84.4 \%)$, married $(52.2 \%)$, had education level through high school $(35.4 \%)$, self-reported brown (61.5\%), earned between five and six minimum wage (42.7\%), were aged between 36 and 45 years $(59.1 \%)$, and were soldiers $(53.1 \%)$. A total of seven (7.3\%) among the study participants had metabolic syndrome. Regarding the components of metabolic syndrome, increased waist circumference indicating risk for cardiovascular disease was identified in $34.4 \%$ of the participants; overweight was identified in $57.3 \%$; hypertension in $11.5 \%$; high triglycerides in $29.5 \%$; decreased level of high-density lipoprotein cholesterol in $60.4 \%$; and high fasting blood glucose in $31.3 \%$. The corporal rank was associated with metabolic syndrome: a military with corporal rank had a risk eleven times higher of having criteria for metabolic syndrome compared to other ranks (odds ratio $11.86 ; 95 \%$ confidence interval 10.88-12.84; $\mathrm{p}=0.005$ ). Conclusions: The military policemen of the investigated sample had a lower prevalence of metabolic syndrome when compared to other Brazilian studies. The corporal rank had a higher risk of developing metabolic syndrome, which should be investigated in more depth by studies that look for the relationship between functional characteristics of each military rank and presence of clinical and laboratory components of metabolic syndrome.

KEY WORDS: metabolic syndrome; obesity; insulin resistance; blood pressure; cardiovascular risk; police officer. 
Abreviaturas: SM, síndrome metabólica; HDL-c, colesterol ligado à lipoproteína de alta densidade.

\section{INTRODUÇÃO}

A Síndrome Metabólica (SM) é um transtorno condicionado por fatores de risco cardiovasculares em geral relacionados à deposição central de gordura e à resistência a insulina. Segundo a Sociedade Brasileira de Cardiologia em sua diretriz sobre Diagnóstico e Tratamento da Síndrome Metabólica, a associação da SM com doença cardiovascular eleva a mortalidade geral em cerca de 1,5 vezes e a cardiovascular em cerca de 2,5 vezes [1].

Para a caracterização da SM, alguns fatores precisam ser considerados. A obesidade central, distribuição da gordura do corpo do tipo androide, representa o aspecto principal da SM. Outros fatores que fazem parte da SM são os altos níveis de glicemia, a hipertensão arterial, o aumento de triglicerídeos e a diminuição do colesterol ligado à lipoproteína de alta densidade (HDL-c), que apresenta efeitos anti-inflamatórios, antioxidantes e vasodilatadores [2].

Segundo a Sociedade Brasileira de Metabologia e Endocrinologia, na SM observa-se a resistência à insulina, exigindo que o pâncreas produza mais quantidade desse hormônio e aumentando o seu nível no sangue. Fatores genéticos, excesso de peso (principalmente na região abdominal) e a ausência de atividade física são associados à resistência à insulina [3].

Seguindo os padrões do National Cholesterol Education Program's Adult Treatment Panel III (NCEP-ATP III), o diagnóstico da SM é feito quando três ou mais dos seguintes fatores de risco estão presentes: obesidade abdominal (cintura com mais de $102 \mathrm{~cm}$ em homens e mais de $88 \mathrm{~cm}$ mulheres); baixo HDL-c (homens menos que $40 \mathrm{mg} / \mathrm{dl}$, mulheres menos do que $50 \mathrm{mg} / \mathrm{dl})$; triglicerídeos elevados $(150 \mathrm{mg} / \mathrm{dl}$ ou mais); pressão arterial alta (sistólica igual ou acima de $130 \mathrm{mmHg}$ ou diastólica igual ou acima de $85 \mathrm{mmHg})$; e glicemia de jejum elevada $(110 \mathrm{mg} / \mathrm{dl}$ ou mais) [4].

De acordo com pesquisa realizada por Minayo et al. [5], a respeito da saúde física e mental de policiais civis e militares do Rio de Janeiro, identificou-se grande frequência de problemas de saúde, incluindo obesidade, hipercolesterolemia, hiperglicemia e várias outras morbidades. Esses problemas podem levar ao afastamento do trabalho por invalidez, em decorrência de agravos ou lesões, antes dos os trinta anos de carreira. Em geral, os policiais que ficam incapacitados, temporária ou permanentemente, são encaminhados para atividades internas e administrativas, muitas vezes com prejuízo à corporação [5].

O presente estudo buscou determinar a prevalência de SM e seus componentes nos membros de uma unidade da Polícia Militar da cidade de Aracaju, Estado de Sergipe, no ano de 2016.

\section{MÉTODOS}

Foi desenvolvido um estudo transversal envolvendo os membros de uma unidade da Polícia Militar no Estado de Sergipe, localizada na capital Aracaju, no período de junho a julho de 2016.

Segundo dados do Instituto Brasileiro de Geografia e Estatística, o município de Aracaju apresentava, no ano de 2015, uma população estimada de 632.744 habitantes, distribuídos em uma área de extensão territorial de aproximadamente, $182 \mathrm{~km}^{2}$ [6]. Essa unidade da Polícia Militar (Batalhão de Choque) tem a missão de realizar o controle de distúrbios civis e o policiamento em eventos especiais, como por exemplo, segurança e reintegração de posse de imóveis ocupados e guarda e escolta de presos de alta periculosidade [7].

Foram selecionados para o estudo todos os policiais ativos da unidade que aceitaram participar da pesquisa, assinaram o termo de consentimento e não estavam em licença, afastamento, férias ou em processo de transferência para outra unidade da polícia. Para a coleta dos dados foram utilizados dois questionários estruturados contendo as seguintes informações: 1) dados socioeconômicos e demográficos: escolaridade, renda mensal, estado civil, cor/raça, idade, sexo, naturalidade; 2) ficha de aferição de medidas: peso, altura, índice de massa corporal (IMC), perímetro abdominal, pressão arterial e variáveis bioquímicas verificadas a partir de exames laboratoriais. A síndrome metabólica foi definida a partir dos critérios definidos pela NCEP-ATP III descritos na introdução.

A coleta dos dados foi feita ao longo de um período de dois meses durante a realização dos exames médicos periódicos. A equipe de pesquisa aplicou os questionários após as consultas médicas de rotina e registrou os dados bioquímicos dos exames laboratoriais apresentados pelos policiais ao médico examinador. Os exames foram solicitados pelo médico da Polícia Militar com 45 dias de 
antecedência em relação à marcação da consulta. Os policiais realizaram os exames em um único laboratório.

Os dados foram armazenados nos programas Epidata 3.1. e Epi Info versão 7.0. Foi conduzida uma análise descritiva para caracterização da população estudada segundo as características sociodemográficas e laborais. Uma análise univariada foi realizada para examinar associações entre as variáveis de interesse, utilizando-se o teste qui-quadrado de Pearson e adotando-se o valor de $\mathrm{p}<0,05$ como estatisticamente significativo.

O presente estudo fez parte de uma pesquisa maior intitulada "Saúde, Estilo de Vida e Qualidade de Vida dos Policiais Militares de Sergipe", a qual foi submetida ao Conselho de Ética em Pesquisa com Seres Humanos da Universidade Tiradentes (número do protocolo 1.388.406), seguindo as especificações da Resolução 466/12 do Conselho Nacional de Saúde do Ministério da Saúde. O estudo garantiu o sigilo e a privacidade das informações dadas pelos participantes. Um sistema de identificação numérica foi criado para garantir a não identificação dos indivíduos nos questionários. Todas as informações foram usadas exclusivamente para atender os propósitos deste estudo. Todos os participantes assinaram o Termo de Consentimento Livre e Esclarecido.

\section{RESULTADOS}

Do total de 115 militares lotados na unidade, $96(83,5 \%)$ participaram da pesquisa. A maior parte dos militares era composta por indivíduos do sexo masculino $(96,6 \%)$, com filhos $(84,4 \%)$, casados $(52,2 \%)$, apresentavam nível de escolaridade até o ensino médio $(35,4 \%)$, autorreferiram-se de cor parda $(61,5 \%)$, ganhavam entre cinco e seis salários mínimos $(42,7 \%)$ e estavam com idade entre 36 e 45 anos (59,1\%) (Tabela 1).

Aplicando os critérios do NCEP-ATP III, sete (7,3\%) participantes tinham SM, caracterizando o risco cardiovascular. Em relação aos componentes da síndrome metabólica, $34,4 \%$ dos militares apresentavam circunferência abdominal dentro do risco aumentado para doenças cardiovasculares, 57,3\% tinham sobrepeso, $11,5 \%$ pressão arterial elevada no momento da coleta, 33,3\% triglicerídeos alterados, $60,4 \%$ HDL-c elevado e $31,3 \%$ glicemia de jejum alterada (Tabela 2).

Foi pesquisada a associação entre os níveis de graduação dos militares (soldado, cabo, sargento, tenente ou capitão) e a síndrome metabólica. Os militares cabos tinham um risco quase onze vezes maior de ter critérios para SM (razão de prevalência 11,86; intervalo de confiança 95\% 10,88-12,84; $\mathrm{p}=0,005)$ (Tabela 3).

Depois de ajustar para a prática de atividade física, a análise revelou que entre os policiais que realizavam atividade mais de três vezes por semana, houve uma redução da ocorrência dos critérios para a SM em $73,0 \%$.

Tabela 1. Distribuição segundo dados sociodemográficos de uma amostra de 96 policiais militares do Batalhão de Choque de Sergipe. Aracaju, SE, 2016.

\begin{tabular}{|c|c|c|}
\hline Variável & $\mathbf{n}$ & $\%$ \\
\hline \multicolumn{3}{|l|}{ Sexo } \\
\hline Feminino & 3 & 3,1 \\
\hline Masculino & 93 & 96,9 \\
\hline \multicolumn{3}{|l|}{ Filhos } \\
\hline Sim & 81 & 84,4 \\
\hline Não & 15 & 15,6 \\
\hline \multicolumn{3}{|l|}{ Situação conjugal } \\
\hline Casado & 50 & 52,1 \\
\hline União estável & 24 & 25,0 \\
\hline Solteiro & 11 & 11,5 \\
\hline Viúvo & 1 & 1,0 \\
\hline Desquitado / divorciado / separado & 10 & 10,4 \\
\hline \multicolumn{3}{|l|}{ Escolaridade } \\
\hline Ensino médio & 34 & 35,4 \\
\hline Ensino superior completo & 24 & 25,0 \\
\hline Ensino superior incompleto & 29 & 30,2 \\
\hline Especialização & 7 & 7,3 \\
\hline Não respondeu & 2 & 2,1 \\
\hline \multicolumn{3}{|l|}{ Cor } \\
\hline Branca & 13 & 13,5 \\
\hline Amarela & 2 & 2,1 \\
\hline Parda & 59 & 61,5 \\
\hline Preta & 20 & 20,8 \\
\hline Não sabe ou não respondeu & 2 & 2,1 \\
\hline \multicolumn{3}{|l|}{ Renda média familiar* } \\
\hline 2 a 4 salários mínimos & 34 & 35,4 \\
\hline 5 a 6 salários mínimos & 41 & 42,7 \\
\hline 7 a 9 salários mínimos & 11 & 11,5 \\
\hline Acima de 10 salários mínimos & 8 & 8,3 \\
\hline Não respondeu & 2 & 2,1 \\
\hline \multicolumn{3}{|l|}{ Faixa etária em anos $(n=93)^{\dagger}$} \\
\hline Até 25 anos & 4 & 4,3 \\
\hline Entre 26 e 35 anos & 22 & 23,7 \\
\hline Entre 36 e 45 anos & 55 & 59,1 \\
\hline Acima de 45 anos & 12 & 12,9 \\
\hline
\end{tabular}

* Salário mínimo no período da pesquisa $=\mathrm{R} \$ 880,00$

+ Para a variável faixa etária três participantes não responderam. 
Tabela 2. Componentes da síndrome metabólica identificados em uma amostra de 96 policiais militares do Batalhão de Choque de Sergipe. Aracaju, SE, 2016.

\begin{tabular}{|c|c|c|c|c|}
\hline & n & $\%$ & Média & Desvio padrão \\
\hline Circunferência abdominal (cm) & & & 94,5 & 10,5 \\
\hline$\leq 102$ & 39 & 40,6 & & \\
\hline$>102$ & 57 & 59,4 & & \\
\hline Índice de massa corporal $\left(\mathrm{kg} / \mathrm{m}^{2}\right)$ & & & 26,8 & 4,4 \\
\hline Eutrófico & 27 & 28,1 & & \\
\hline Sobrepeso & 55 & 57,3 & & \\
\hline Obesidade & 14 & 14,6 & & \\
\hline Pressão arterial (mmHg) & & & $119 \times 78$ & $\mathrm{PAS}=33,3$ \\
\hline Normal & 84 & 87,5 & & $\mathrm{PAD}=22,3$ \\
\hline Hipertensão & 11 & 11,5 & & \\
\hline Triglicerídeos (mg/dL) & & & 126,7 & 79,9 \\
\hline$<150$ & 64 & 66,7 & & \\
\hline$\geq 150$ & 32 & 33,3 & & \\
\hline HDL-c (mg/dL) & & & 43,7 & 16,01 \\
\hline$<40 \mathrm{mg} / \mathrm{dl}$ & 38 & 39,6 & & \\
\hline$\geq 40 \mathrm{mg} / \mathrm{dl}$ & 58 & 60,4 & & \\
\hline Glicemia de jejum (mg/dL) & & & 113,9 & 130,7 \\
\hline Normal & 66 & 68,8 & & \\
\hline Alterada & 30 & 31,3 & & \\
\hline
\end{tabular}

PAS, pressão arterial sistólica; PAD, pressão arterial diastólica; HDL-c, colesterol ligado à lipoproteína de alta densidade.

Tabela 3. Distribuição de uma amostra de $87^{*}$ policiais militares do Batalhão de Choque de Sergipe segundo níveis de graduação e prevalência de síndrome metabólica. Aracaju, SE, 2016.

\begin{tabular}{lcccc}
\hline \multicolumn{1}{c}{ Graduação } & \multicolumn{2}{c}{$\begin{array}{c}\text { Número } \\
\text { total }\end{array}$} & \multicolumn{2}{c}{$\begin{array}{c}\text { Com síndrome } \\
\text { metabólica }\end{array}$} \\
\cline { 2 - 3 } & $\mathbf{n}$ & $(\%)$ & & $\mathbf{n}$ \\
\hline Cabo & 23 & $(26,4)$ & 5 & $(71,4)^{\dagger}$ \\
Capitão & 1 & $(1,1)$ & 0 & $(0,0)$ \\
Sargento & 11 & $(12,6)$ & 0 & $(0,0)$ \\
Soldado & 51 & $(58,6)$ & 2 & $(28,6)$ \\
Tenente & 1 & $(1,1)$ & 0 & $(0,0)$ \\
\hline
\end{tabular}

* Para esta variável, nove policiais não responderam.

A graduação de soldado foi tomada como grupo de referência.

+ Teste do qui-quadrado de Pearson. Razão de prevalência = 11,86; intervalo de confiança $95 \%=10,88-12,84 ; p=0,005$.

Após ajuste para a prática de atividade física: razão de prevalência $=11,13$; intervalo de confiança $95 \%=10,29-11,97 ; p=0,004$.

\section{DISCUSSÃO}

Analisando as pesquisas sobre SM entre policiais militares, percebe-se que, no Brasil, apesar da crescente ocorrência de estudos com essa população, ainda são escassos os estudos abordando esta temática, sobretudo que envolvam a região nordeste do país. Este estudo mostra que a maioria dos militares era do sexo masculino, corroborando com grande parte dos estudos com policiais, confirmando a realidade brasileira de predominância de homens nessa profissão. Neste estudo, a maioria dos participantes estava acima dos 35 anos de idade. Pesquisas revelam uma progressão linear da prevalência da SM com a idade. A organização do trabalho militar pode favorecer a ocorrência de doenças metabólicas em virtude de características da própria profissão, relacionadas a estresse e outras interferências do trabalho sobre o estilo de vida [8-15].

Entretanto, o presente estudo identificou uma prevalência baixa da SM entre os participantes, em comparação com outros trabalhos realizados com militares. Um estudo realizado com policiais militares no sul de Minas Gerais identificou uma prevalência de 21,0\%. Na Paraíba, foi realizado um estudo também com militares, e a prevalência foi de $17,4 \%$; e no Distrito Federal, outra pesquisa encontrou 16,5\%, demonstrando uma variação que pode depender de características de vida e de trabalho, ou mesmo dos métodos de coleta de dados de cada estudo [9-11]. Nesta amostra de Aracaju, Sergipe, a baixa prevalência da SM pode ser justificada pela realização de atividade física associada a um consumo de frutas e verduras em mais de três vezes por semana entre os militares incluídos, fatores que favorecem a diminuição da ocorrência de alterações metabólicas e bioquímicas.

A rotina desses profissionais apresenta-se geralmente muito desgastante em virtude das características do trabalho, como horários, locais e refeições 
inadequadas, fatores estes que podem ter influência no desencadeamento de alterações hematológicas e antropométricas. Ao comparar os valores obtidos das medidas de circunferência abdominal, por exemplo, verificou-se que o percentual obtido entre os militares com risco aumentado para doenças cardiovasculares foi semelhante ao de outros estudos. Ressalta-se que o número de indivíduos nesta pesquisa com obesidade central foi o equivalente a $25 \%$ da tropa, configurando assim um alerta substancial e importante para intervenções imediatas nessa população, a fim de prevenir complicações cardiovasculares e metabólicas [12,13].

Quanto à obesidade, nesta amostra observou-se um percentual próximo ao encontrado em um estudo italiano, em que a prevalência de sobrepeso e obesidade superou os 50\% [14]. Fato preocupante, uma vez que a obesidade traz consequências graves ao sistema cardiovascular. Situações como trabalho noturno, carga horária elevada e revezamento de turnos podem contribuir para a privação do sono e alimentação em horários irregulares, favorecendo assim o ganho excessivo de peso.

Essas características ocupacionais também contribuem para a ocorrência de dislipidemia e aumento da pressão arterial. Apesar de que no presente estudo a prevalência de hipertensão arterial foi baixa em relação a outros estudos, é necessário voltar-se para esse dado, no qual é possível intervir preventivamente. A hipertensão é tida como um fator de risco para diversas morbidades, entre elas as cardiovasculares, que também são evidenciadas e provocadas pela alteração nos níveis de lipídeos, como o HDL-c baixo e triglicerídeos elevados. Estes são tidos como principais determinantes da ocorrência de doenças cardiovasculares e cerebrovasculares, dentre elas aterosclerose, infarto agudo do miocárdio, doença isquêmica do coração e acidente vascular cerebral [15-17].

Estudos nacionais e internacionais mostram variações na prevalência de níveis de glicemia elevados (observaram-se percentuais de 10,5\% até 56\%). Em Sergipe, o percentual de policiais com glicemia elevada, que pode sugerir resistência insulínica, foi de $31,3 \%$, indicador de alerta para intervenções coletivas na corporação. $\mathrm{O}$ diabetes mellitus tipo 2 pode ser evitável em muitos casos [18-23].

A necessidade de ações e estratégias voltadas para a prevenção da SM e a mudança de hábitos alimentares e de nível de atividade física entre os que já apresentam a síndrome são ações importantes e não devem ser apenas individuais, mas também coletivas, envolvendo instituições e políticas públicas. Entre as ações que poderiam ser implementadas na Polícia Militar, estaria a abordagem da SM por intermédio de um serviço especializado em engenharia de segurança e medicina do trabalho, que trabalharia com medidas de prevenção primária e promoção da saúde a partir de um programa de controle médico de saúde ocupacional, incluindo a inserção de intervenções sistematizadas voltadas para os mais relevantes agravos da SM.

Vale considerar os fatores limitantes deste estudo como pontos importantes que devem ser sinalizados nesta discussão. $\mathrm{O}$ estudo transversal pode trazer um viés de prevalência, já que, por mostrar a imagem instantânea do objeto do estudo, podem ser identificados somente os sobreviventes ao efeito estudado e sua situação quanto à exposição presente. Isso pode explicar a prevalência global de SM mais baixa em relação a outros estudos ocupacionais [24]. O segundo ponto diz respeito ao efeito do trabalhador sadio; é a situação particular do tipo de estudo em epidemiologia ocupacional que exclui o possível doente. Os policiais analisados estavam, no momento da pesquisa, em seu local de trabalho. Essa situação pode subestimar o tamanho dos riscos oferecidos pelo processo de trabalho, pois os mais afetados não conseguem se manter em atividade, afastando-se por licenças médicas ou outros motivos. Assim, de qualquer forma o resultado obtido é preocupante, por esses indivíduos podem agravar seu estado de saúde e gerar grandes prejuízos para a qualidade do seu trabalho, com consequências importantes na execução da segurança pública $[25,26]$.

Percebeu-se que a realização rotineira de atividade física influenciou na diminuição da ocorrência dos fatores para a síndrome. Diante disto, recomenda-se um acompanhamento dos policiais, no que tange ao incentivo e realização da prática de exercícios físicos, além de intervir com ações educativas voltadas para mudanças de hábitos de vida e controle dos fatores de risco que se apresentaram com valores limítrofes entre os profissionais.

Conclui-se que os policiais aracajuanos da amostra investigada apresentaram uma prevalência de síndrome metabólica menor quando comparada a outros estudos brasileiros, sendo que a graduação de cabo apresentou maior risco de desenvolver síndrome metabólica, o que deve ser investigado com mais profundidade por meio de estudos que relacionem as características funcionais de cada patente com a presença dos componentes clínicos e laboratoriais da síndrome metabólica. Observa-se também que são necessárias mais pesquisas para explorar a fisiopatologia da síndrome metabólica associada a características de trabalho no grupo dos policiais militares. 


\section{NOTA}

Declaração de conflitos de interesse

Os autores declaram não haver conflitos de interesse relevantes ao conteúdo deste estudo.

\section{REFERÊNCIAS}

1. I Diretriz Brasileira de Diagnóstico e Tratamento da Síndrome Metabólica. Arq Bras Cardiol. 2005;84:1-27. http://dx.doi.org/10.1590/ S0066-782X2005000100001

2. Ferrari, CKB. Atualização: fisiopatologia e clínica da síndrome metabólica. Arq Cat Med. 2007;36(4):90-5.

3. Godoy-Matos AF, Oliveira J, Guedes EP, Carraro L, Lopes AC, Mancini MC, Suplicy HL, Brito CLS, Bystronski DP, Mombach KD, Stenzel LM, Repetto G, Radominski RB, Halpern ZSC, Villares SMF, Arrais RF, Rodrigues MDB, Mazza FC, Bittar T, Benchimol AK; Associação Brasileira para o Estudo da Obesidade e da Síndrome Metabólica (Abeso). Diretrizes Brasileiras de Obesidade. $3^{\mathrm{a}}$ ed. São Paulo: AC Farmacêutica; 2009

4. Gottlieb MGV. Origem da síndrome metabólica: aspectos genético-evolutivos e nutricionais. Sci Med. 2008;18(1):31-8.

5. Minayo MCS, Assis SG, Oliveira RVC. Impacto das atividades profissionais na saúde física e mental dos policiais civis e militares do Rio de Janeiro (RJ, Brasil). Ciênc Saúde Coletiva. 2011;16(4):2199-209. http://dx.doi.org/10.1590/S1413-81232011000400019

6. Instituto Brasileiro de Geografia e Estatística (IBGE). Cidades: Sergipe - informações básicas dos municípios. 2015 [cited 2016 ]. Available from: http://www.cidades.ibge.gov.br/xtras/uf.php?lang=\&coduf=28\&search=sergipe

7. Sergipe. Secretaria de Segurança Publica de Sergipe (SSP/SE). Histórico do Batalhão de Choque da Policia de Sergipe [Internet]. Aracaju; 2014 [cited 2016 Oct 05]. Available from: http://www.pm.se.gov.br/institucional/historico/

8. Oliveira PLM, Bardagi MP. Estresse e comprometimento com a carreira em policiais militares. Bol Psicol. 2009; 59(131):153-66.

9. Vilarinho RMF, Lisboa, MTL. Diabetes mellitus: fatores de risco em trabalhadores de enfermagem. Acta Paul Enferm. 2010;23(4):557-61. http://dx.doi.org/10.1590/S0103-21002010000400018

10. Jesus GM, Jesus EFA. Predisposição para desenvolver resistência insulínica em policiais militares. Pensar Prat. 2010;13(2):1-15.

11. Tahan F, Pereira JC. Avaliação de risco cardiovascular por indicadores antropométricos em policiais militares de um batalhão do Sul de Minas Gerais. Rev Bras Gastroenterol. 2015;14(4):230-6.

12. Cordeiro AKR. Avaliação da Síndrome Metabólica em Policiais Militares do Segundo Batalhão de Policia Militar da Paraíba [monografia]. [João Pessoa]: Universidade Federal da Paraíba; 2015. 27 p.

13. Leite EB, Anchieta VCC. Identificação de Síndrome Metabólica em Policiais Civis do Distrito Federal. Brasília Med. 2013;50(3):186-93. http://dx.doi.org/10.14242/2236-5117.2014v50n3a88p186

14. Garbarino S, Magnavita N. Work stress and metabolic syndrome in police officers. a prospective study. PLoS One. 2015 Dec 7;10(12):e0144318. http://dx.doi.org/10.1371/journal.pone.0144318

15. Oliveira AF. Nutrição e síndrome metabólica em policiais militares do oeste do Paraná [tese]. [São Paulo]: Universidade Federal de São Paulo (UNIFESP); 2009. 118 p.

16. Barbosa RO, Silva EF. Prevalência de fatores de risco cardiovascular em policiais militares. Rev Bras Cardiol. 2013;26(1):45-53.

17. Oliveira CM, Cruz MM. Sistema de vigilância em saúde no Brasil: avanços e desafios. Saúde Debate. 2015;39(104):255-67. http://dx.doi. org/10.1590/0103-110420151040385

18. Rocha RM, Barra GB, Campos EC, Rosa C, Garcia EC, Amato AA, Azevedo MF. Prevalence of the rs 1801282 single nucleotide polymorphism of the PPARG gene in patients with metabolic syndrome. Arch Endocrinol Metab. 2015 Aug;59(4):297-302. http://dx.doi. org/10.1590/2359-3997000000086

19. Braga Filho RT, D'Oliveira A. The Prevalence of Metabolic Syndrome Among Soldiers of the Military Police of Bahia State, Brazil. Am J Mens Health. 2014 Jul;8(4):310-5. http://dx.doi.org/10.1177/1557988313510928

20. Wirth MD, Burch J, Shivappa N, Violanti JM, Burchfiel CM, Fekedulegn D, Andrew ME, Hartley TA, Miller DB, Mnatsakanova A, Charles LE, Steck SE, Hurley TG, Vena JE, Hébert JR. Association of a dietary inflammatory index with inflammatory indices and metabolic syndrome among police officers. J Occup Environ Med. 2014 Sep;56(9):986-9. http://dx.doi.org/10.1097/JOM.0000000000000213

21. Lucena MM. Risco cardiovascular em policiais e bombeiros de Campina Grande - Paraíba. [monografia]. [João Pessoa]: Universidade Federal da Paraíba; 2014. 33 p.

22. Bello-Rodriguez BM, Sanchez-Cruz G, Delgado-Bustillo F, Asiama G. The relationship between metabolic syndrome and target organ damage in Ghanaian with stage-2 hypertension. Ghana Med J. 2013 Dec;47(4):189-96.

23. Hartley TA, Burchfiel CM, Fekedulegn D, Andrew ME, Knox SS, Violanti JM. Associations between police officer stress and the metabolic syndrome. Int J Emerg Ment Health. 2011;13(4):243-56.

24. Batista UM. Prevalência de sobrepeso, obesidade e Fatores de Risco para doenças cardiovasculares em policiais militares masculinos efetivos de Goiânia - GO [dissertação]. [Goiania]: Universidade de Trás-Os-Montes e Alto Douro; 2011. 153 p.

25. Fonseca ISS, Araújo TM. Prevalência de transtornos mentais comuns entre indústriarios bahianos. Rev Bras Saúde Ocup. 2014;39 (129): 35-49. http://dx.doi.org/10.1590/0303-7657000065012

26. Carlotto, MS. Síndrome de Burnout em professores: prevalência e fatores associados. Psic Teor e Pesq. 2011;27(4):403-10. http://dx.doi. org/10.1590/S0102-37722011000400003 